\title{
Infusion-Compatible Antibiotic Formulations for Rapid Administration to Improve Outcomes in Cancer Outpatients With Severe Sepsis and Septic Shock: The Sepsis STAT Pack
}

\author{
Jason D. Goldman, MD, MPH ${ }^{a, b, c, d}$; Amelia Gallaher, BS ; Rupali Jain, PharmD; Zach Stednick, MPH; \\ Manoj Menon, MD, MPH ${ }^{\mathrm{a}, \mathrm{c}, \mathrm{g}}$; Michael J. Boeckh, MD, PhD ${ }^{\mathrm{a}, \mathrm{b}, \mathrm{c}}$; Paul S. Pottinger, MD ${ }^{\mathrm{b}, \mathrm{c}}$; \\ Stephen M. Schwartz, PhD ${ }^{\mathrm{d}, \mathrm{h}}$; and Corey Casper, MD, MPH ${ }^{\mathrm{a}, \mathrm{b}, \mathrm{c}, \mathrm{d}, \mathrm{h}}$
}

\begin{abstract}
Background: Patients with cancer are at high risk for severe sepsis and septic shock (SS/SSh), and a delay in receiving effective antibiotics is strongly associated with mortality. Delays are due to logistics of clinic flow and drug delivery. In an era of increasing antimicrobial resistance, combination therapy may be superior to monotherapy for patients with SS/SSh. Patients and Methods: At the Seattle Cancer Care Alliance, we implemented the Sepsis STAT Pack (SSP) program to simplify timely and effective provision of empiric antibiotics and other resuscitative care to outpatients with cancer with suspected SS/SSh before hospitalization. Over a 49-month period from January 1, 2008, through January 31, 2012, a total of 162 outpatients with cancer received the intervention. A retrospective cohort study was conducted to determine outcomes, including mortality and adverse events associated with the use of a novel care bundle designed for compatibility of broad-spectrum antibiotics and other supportive care administered concurrently via rapid infusion at fixed doses. Results: Of 162 sequential patients with cancer and suspected SS/SSh who received the SSP, $71(44 \%)$ were diagnosed with SS/SSh. Median age was 53 years and $65 \%$ were men; $141(87 \%)$ had hematologic malignancies, 77 (48\%) were transplant recipients, and 80 (49\%) were neutropenic. Median time to completion of antibiotics was 111 minutes (interquartile range, $60-178$ minutes). A total of 71 patients (44\%) had bacteremia and $17 \%$ of 93 isolates were multidrug-resistant. Possibly related nephrotoxicity occurred in 7 patients, and 30-day mortality occured in 6 of 160 patients (4\%), including 3 of $71(4 \%)$ with SS/SSh. Risk of developing SSh or death within 30 days increased $18 \%(95 \% \mathrm{Cl}, 4 \%-34 \%)$ for each hour delay to completion of antibiotics $(P=.01)$. Conclusions: Rapidly administered combination antibiotics and supportive care delivered emergently to ambulatory patients with cancer with suspected SS/SSh was well-tolerated and associated with excellent short-term survival.
\end{abstract}

J Natl Comp Canc Netw 2017;15(4):457-464

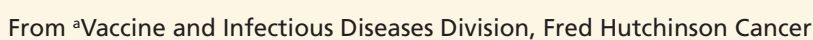

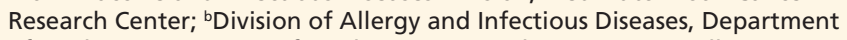
of Medicine, University of Washington; 'Seattle Cancer Care Alliance; ${ }^{d}$ Department of Epidemiology, School of Public Health, University of Washington; eschool of Medicine, University of Washington; 'School of Pharmacy, University of Washington; 9Division of Hematology, Department of Medicine, University of Washington; and hPublic Health Sciences Division, Fred Hutchinson Cancer Research Center, Seattle, Washington. Submitted July 29, 2016; accepted for publication December 8, 2016. Dr. Boeckh has disclosed that he has received research support and served as a consultant for Merck and GlaxoSmithKline. Dr. Casper has disclosed that he serves on an external advisory board for and receives travel compensation from GlaxoSmithKline and receives research support from Janssen. The remaining authors have disclosed that they have no financial interests, arrangements, affiliations, or commercial interests with the manufacturers of any products discussed in this article or their competitors.

This work was supported by the National Institute of Allergy and Infectious Diseases (T32 A10740) and National Center for Advancing
Translational Sciences (UL1TR000423), both at the National Institutes of Health. This work was also supported by a grant from the Division of Allergy and Infectious Disease at the University of Washington (J.D.G.). The content is solely the responsibility of the authors and does not necessarily represent the official views of the NIH. The sponsors had no role in the design and conduct of the study; collection, management, analysis, and interpretation of the data; and preparation, review, or approval of the manuscript.

Author contributions: Clinical program design: Casper, Boeckh. Study design: Goldman, Pottinger, Schwartz, Casper. Acquisition of data: Goldman, Gallaher, Jain, Stednick. Analysis of data: Goldman. Interpretation of data: Goldman, Jain, Menon, Boeckh, Pottinger, Schwartz, Casper. Drafting of manuscript: Goldman. Critical revision and final approval: all authors.

Correspondence: Jason D. Goldman, MD, MPH, Fred Hutchinson Cancer Research Center, 1100 Fairview Avenue North, Box M1-B140, Seattle, WA 98109. E-mail: jdgold@uw.edu 
Patients with cancer are at high risk for death from severe sepsis and septic shock (SS/SSh), especially those with disease-related or iatrogenic immunosuppression. ${ }^{1,2}$ Mortality due to sepsis is poorly studied in patients with cancer in the ambulatory setting, where most cancer care is delivered in the United States. Delay to effective antimicrobial therapy is strongly associated with mortality, ${ }^{3-6}$ such that a low threshold for intervention is required before a full clinical assessment. Choice of empiric antibiotics must balance the increased toxicity of combination therapy ${ }^{7}$ with the survival benefit of antibiotics with an appropriate spectrum of activity. ${ }^{4}$ Multiple national guidelines ${ }^{8,9}$ strongly recommend monotherapy for uncomplicated neutropenic fever, but differ regarding which antibiotics should be included in combination therapy for initial management of SS/SSh, reflecting sparse and conflicting data in patients with cancer and SS/SSh.

We reasoned that intervening in the disease process of patients with cancer and SS/SSh using an aggressive approach prioritizing completion of broad antibiotics should improve overall mortality. Many antibiotic combinations require long infusion times and are incompatible for coadministration (ie, vancomycin, gentamicin); these logistics of drug delivery lead to delays in completion of effective antimicrobial therapy. The Sepsis STAT Pack (SSP) program simplifies emergent administration of broad-spectrum antibiotics that can be coadministered via rapid infusion. The protocol ensures at least one active agent is delivered in this heavily antibiotics-treated population with a high prevalence of antibiotic resistance. ${ }^{10}$ We report on the feasibility, mortality, and adverse events associated with the use of this novel care bundle among a unique cohort of ambulatory patients with cancer and suspected SS/SSh.

\section{Patients and Methods}

\section{Patients and Study Setting}

The Seattle Cancer Care Alliance (SCCA) is a care consortium and includes Fred Hutchinson Cancer Research Center (FHCRC), University of Washington (UW), and Seattle Children's Hospital. Patients are predominantly seen at the outpatient SCCA clinic, including those receiving specialized care, such as hematopoietic stem cell transplantation (HSCT). These ambulatory patients are highly im- munosuppressed, including deep and prolonged neutropenia, and often require intensive therapies, such as daily infusions or transfusion support. ${ }^{11}$ Adults requiring hospitalization are transferred by ambulance to the UW Medical Center, located 3 miles from the SCCA clinic.

The SSP is a clinical algorithm developed to simplify the emergent administration of broad-spectrum antibiotics and other supportive care for outpatients with cancer and presumed SS/SSh before hospital transfer. The goal of the SSP program was to rapidly administer antibiotics with a broad antimicrobial spectrum inclusive of resistant organisms typically encountered in patients with cancer. To reduce administration time, the antibiotic regimen was chosen to be compatible for coadministration via rapid infusion (within 30 minutes) in fixed doses through a single intravenous line. The regimen includes an antipseudomonal carbapenem (or aztreonam for penicillin allergy), aminoglycoside (tobramycin), and oxazolidinone (linezolid), stored as a ready kit in the pharmacy. A preprinted order form that includes reminders of SS/SSh criteria was designed to trigger delivery of antibiotics and other supportive care measures, such as infusion of crystalloids, administration of stress-dose steroids, laboratory tests, blood cultures before administration of antibiotics, and arrangement of rapid transport for hospital admission.

Outpatients with cancer at the SCCA clinic with hematologic or solid malignancies were prospectively treated if presumptively diagnosed with SS/SSh. Patients were included in this cohort if 3 classes of antibiotics were prescribed for the treatment of presumed SS/SSh; exclusion criteria included age $<18$ years or receipt of $<3$ antibiotic classes without indication of rationale. Data were extracted from electronic databases or manually abstracted by 2 reviewers (J.D.G., A.G.; see supplemental eAppendix 1, available with this article at JNCCN.org). This study was approved by the FHCRC Institutional Review Board.

\section{Definitions}

The Surviving Sepsis Campaign criteria were used to define the sepsis disease severity. ${ }^{12}$ Briefly, systemic inflammatory response syndrome (SIRS) was diagnosed given $\geq 2$ signs of systemic inflammation, sepsis was diagnosed given SIRS and suspected or confirmed infection, SS was diagnosed given sepsis and 
organ dysfunction or tissue hypoperfusion, and SSh was diagnosed given SS and persistent hypotension despite adequate fluid challenge or need for vasopressors. A conservative approach to ascribing physiologic derangements to the sepsis syndrome was adopted (supplemental eAppendix 1) due to frequent hematologic and physiologic abnormalities arising from the underlying malignancy or related therapies. Hypotension determined on repeated measurements and temporally related to infection was considered criteria for SS/SSh.

Neutropenia was defined as an absolute neutrophil count $\leq 500$ cells/mcL. ${ }^{8}$ Severity of illness was estimated using APACHE II score. ${ }^{13}$ Bloodstream isolates were summarized as sensitive, drug-resistant (DR), multidrug-resistant (MDR), or extensively DR (XDR) according to guidelines. ${ }^{14,15}$ Acute kidney injury (AKI) was defined as an increase in serum creatinine from prior $>0.5 \mathrm{mg} / \mathrm{dL} .{ }^{12}$ Nephrotoxicity was determined to be "definitely related" if AKI followed aminoglycoside therapy without alternative causes; "possibly related" if concurrent nephrotoxic therapies were being given or hypotension was present; and "unrelated" if AKI preceded receipt of aminoglycoside and creatinine subsequently normalized.

\section{Statistical Analysis}

Patients were censored on the last recorded clinical visit or date of death, ascertained from 2 independent sources (supplemental eAppendix 1). For each 1-hour delay from start of clinical encounter to completion of the third antibiotic, a generalized linear regression model was specified with a Poisson distribution and a log-link function using robust standard error to measure relative risk of (1) cumulative 30-day mortality, (2) development of SSh, or (3) the combined outcome of cumulative 30-day mortality or development of SSh. Multivariate models were constructed to control for prespecified confounding variables associated at $P<.20$ level (eTables 1 and 2 ) with both the exposure and the outcome in a parsimonious model and with either the exposure or the outcome in a maximally adjusted model.

To determine efficacy of the SSP antibiotics in the absence of a control group, actual antimicrobial susceptibility was compared with "hypothetical alternative scenarios." Isolated bloodstream bacteria were recategorized as susceptible or resistant if antibiotics were administered per the Infectious Diseases Society of America (IDSA) guidelines for neutropenic fever. ${ }^{8}$ In these scenarios, monotherapy with intravenous antipseudomonal $\beta$-lactam would be administered for uncomplicated neutropenic fever, and glycopeptide (vancomycin) would be added if indicated for hemodynamic instability, pneumonia, or skin/soft tissue infections. The proportions of resistant microbes to the observed and alternative scenarios were compared with McNemar test using the bacterial isolate as the unit of comparison.

Summary data are presented as counts and percentages, means with standard deviations (SD), medians and interquartile ranges (IQR), or proportions with 95\% confidence intervals (95\% CI). Chisquare tests for equal proportion, $t$-tests, or rank sum tests (Wilcoxon, Kruskal-Wallis) were used to test univariate associations. Data analysis was performed using Stata 13.1 software (StataCorp LP, College Station, TX).

\section{Results}

\section{Study Participants}

From January 1, 2008, through January 31, 2012, a total of 162 outpatients with cancer met inclusion criteria (Figure 1). The median age was 53 years (IQR, 42-63 years), 106 (65\%) were men, and 127 (78\%) were Caucasian, 8 (5\%) black, 8 (5\%) Asian, 6 (4\%) Hispanic, and 13 (8\%) other/not reported. Hematologic malignancies accounted for the underlying oncologic diagnosis in 141 patients (87\%), 77 (48\%) had

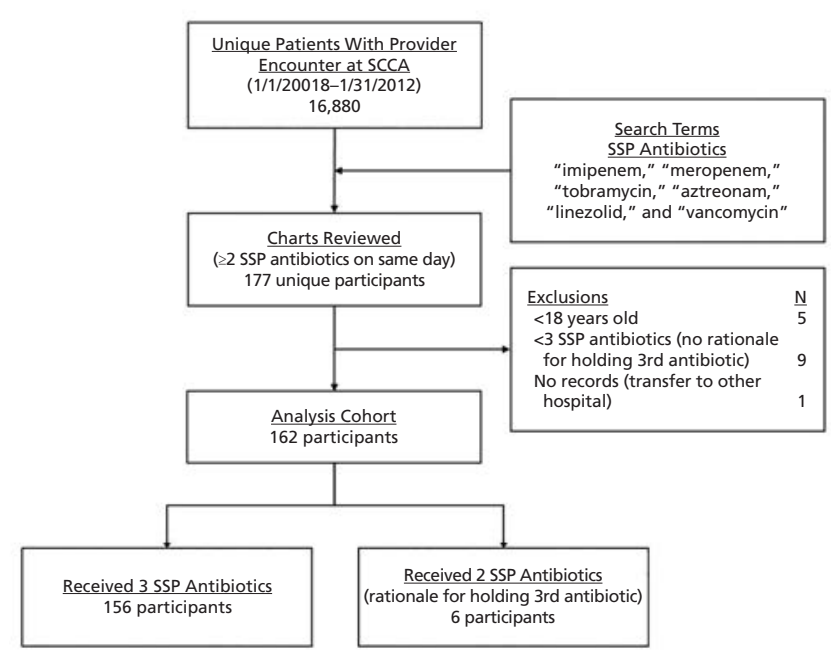

Figure 1. SSP cohort identification flow diagram. Abbreviations: SCCA, Seattle Cancer Care Alliance; SSP, Sepsis STAT Pack. 
received prior HSCT, and 80 (49\%) were neutropenic. At the time of SSP administration, 45 patients (28\%) were on immunosuppressive therapy, and prophylactic antibiotics were prescribed in 105 (65\%; Table 1).

\section{Processes of Care}

SSP antibiotics were administered with bundled resuscitation. Primary antipseudomonal $\beta$-lactam antibiotics were imipenem in 107 patients $(66 \%)$, meropenem in $27(17 \%)$, aztreonam in $24(15 \%)$, and ceftazidime in $4(2 \%)$; second gram-negative coverage was tobramycin in 153 patients (94\%), gentamicin in $2(1 \%)$, and levofloxacin in $1(1 \%)$,

\begin{tabular}{|c|c|c|}
\hline Clinical Characteristics & $\mathrm{N}(\%)^{\mathrm{a}}$ & n (\%) \\
\hline Oncologic diagnosis & $162(100 \%)$ & \\
\hline Leukemia & & $67(41.4 \%)$ \\
\hline Lymphoma & & $48(29.6 \%)$ \\
\hline Multiple myeloma & & $26(16.0 \%)$ \\
\hline Myelodysplastic syndromes & & $11(6.8 \%)$ \\
\hline Solid tumors & & $6(3.7 \%)$ \\
\hline Otherc & & $4(2.5 \%)$ \\
\hline Received HSCT & $77(47.5 \%)$ & \\
\hline Allogeneic & & $50(64.9 \%)$ \\
\hline Autologous & & $27(35.1 \%)$ \\
\hline Neutropenia & $80(49.4 \%)$ & \\
\hline Immunosuppressive therapy & $45(27.8 \%)$ & \\
\hline Tacrolimus & & $21(46.7 \%)$ \\
\hline MMF & & $18(40.0 \%)$ \\
\hline Cyclosporin & & $18(40.0 \%)$ \\
\hline Sirolimus & & $2(4.4 \%)$ \\
\hline Prednisone $(\geq 20 \mathrm{mg} / \mathrm{d}$ ) & & $16(35.6 \%)$ \\
\hline Antibiotics & $105(64.8 \%)$ & \\
\hline Levofloxacin & & $45(41.9 \%)$ \\
\hline TMP-SMX & & $39(37.1 \%)$ \\
\hline Dapsone & & $9(8.6 \%)$ \\
\hline Other & & $34(32.4 \%)$ \\
\hline
\end{tabular}

Abbreviations: HSCT, hematopoietic stem cell transplant; MMF, mycophenolate mofetil; TMP-SMX, trimethoprim-sulfamethoxazole. acCategory data given as N (\%) and data available on all 162 participants.

bsubcategory data given as $\mathrm{n}(\%)$ are not mutually exclusive for immunosuppressives and antibiotics.

Includes aplastic anemia $(n=2)$, lymphoproliferative disease, not otherwise specified $(n=1)$, and hemophagocytic lymphohistiocytosis $(H L H ; n=1)$. and a second agent was held in 6 ( 3 for AKI, 1 for baseline hearing loss, 1 for pediatric protocol, and 1 for allergy). Gram-positive coverage included linezolid in 154 patients (95\%), vancomycin in $6(4 \%)$, and daptomycin in $1(1 \%)$. Dexamethasone as stressdose steroids was administered to 74 patients (46\%) and crystalloid intravenous fluids were started in clinic in 148 of 149 patients (99\%). All patients had same-day blood cultures drawn and 148 of 160 (93\% CI, 87\%-96\%) had blood cultures drawn before antibiotics, with median time before antibiotics of 40 minutes (IQR, 15-81 minutes).

Completion of antibiotic infusions within 1 hour of presentation to the clinic was achieved for 41 patients $(26 \%)$, within 2 hours for 88 (55\%), within 3 hours for 123 (77\%), within 4 hours for 143 $(89 \%)$, and $>4$ hours for $17(11 \%)$ (Figure 2$)$. Time from clinical encounter to antibiotic dispensing accounted for the longest delays (eTable 3). Only 6 patients were not directly admitted to the hospital; 4 of these returned for admission, 3 of whom developed SS/SSh by hospital admission.

Hospital teams empirically de-escalated antibiotics to a 1 - or 2-drug regimen. In 156 admitted patients, tobramycin and linezolid were continued past hospital day 1 (HD1) in only 12 $(8 \%)$ and 13 patients (8\%), respectively. De-escalation of carbapenem or monobactam was less regularly achieved; by HD4, 104 of 156 patients $(67 \%)$ were de-escalated to an intravenous antipseudomonal cephalosporin and 71 of 156 (46\%) were de-escalated to early-generation cephalosporin or quinolone. Of the 85 patients continuing

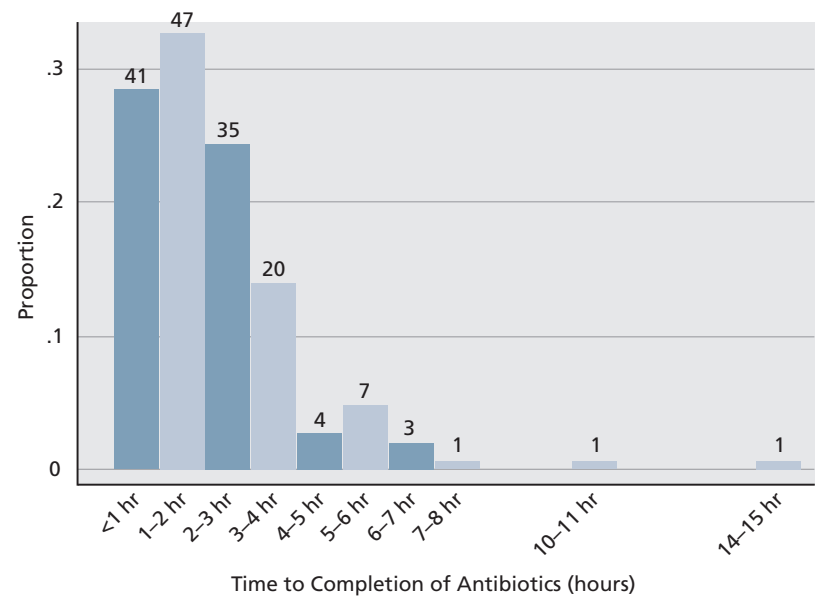

Figure 2. Histogram of time from start of clinical encounter to completion of the third Sepsis STAT Pack antibiotic. 
Sepsis STAT Pack for Patients With Cancer

\begin{tabular}{|c|c|c|c|c|c|}
\hline $\begin{array}{l}\text { Disease } \\
\text { Category }\end{array}$ & $\begin{array}{l}\text { Patients, } \\
\text { N (\%) }\end{array}$ & Neutropenia, n (\%) & Diagnosis $^{\mathrm{b}}$ & $n(\%)$ & $\begin{array}{l}\text { 30-Day Mortality, n (\%) } \\
\text { [Missing] }\end{array}$ \\
\hline No SIRS & $13(8.0)$ & $7(53.9)$ & $\begin{array}{l}\text { Bacteremia } \\
\text { Other infection } \\
\text { Suspected infection } \\
\text { Alternative diagnosis }\end{array}$ & $\begin{array}{l}6(46.2) \\
3(23.1) \\
3(23.1) \\
1(7.7)\end{array}$ & $\begin{array}{r}0(0) \\
{[0]}\end{array}$ \\
\hline SIRS only & $6(3.7)$ & $2(33.3)$ & Alternative diagnosis & $6(100)$ & $\begin{array}{r}0(0) \\
{[1]}\end{array}$ \\
\hline Sepsis & $72(43.8)$ & $40(56.3)$ & $\begin{array}{l}\text { Bacteremia } \\
\text { Other infection } \\
\text { Cx-neg sepsis }\end{array}$ & $\begin{array}{l}24(33.3) \\
19(25.4) \\
29(40.8)\end{array}$ & $\begin{array}{r}3(4.2) \\
{[1]}\end{array}$ \\
\hline Severe sepsis & $46(29.0)$ & 19 (41.3) & $\begin{array}{l}\text { Bacteremia } \\
\text { Other infection } \\
\text { Cx-neg sepsis }\end{array}$ & $\begin{array}{r}29(63.0) \\
4(10.4) \\
13(29.2)\end{array}$ & $\begin{array}{r}2(4.3) \\
{[0]}\end{array}$ \\
\hline Septic shock & $25(15.4)$ & $12(48.0)$ & $\begin{array}{l}\text { Bacteremia } \\
\text { Other infection } \\
\text { Cx-neg sepsis }\end{array}$ & $\begin{array}{r}12(48.0) \\
7(29.2) \\
6(20.8)\end{array}$ & $\begin{array}{r}1(4.0) \\
{[0]}\end{array}$ \\
\hline Total & $162(100)$ & $80(49.4)$ & $\begin{array}{l}\text { Bacteremia } \\
\text { Other infection } \\
\text { Cx-neg sepsis } \\
\text { Suspected infection } \\
\text { Alternative diagnosis }\end{array}$ & $\begin{array}{c}71(43.8) \\
33(20.4) \\
48(29.6) \\
3(1.9) \\
7(4.3)\end{array}$ & $\begin{array}{r}6(3.8) \\
{[2]}\end{array}$ \\
\hline
\end{tabular}

Abbreviations: CX-neg, culture-negative; SIRS, systemic inflammatory response syndrome.

apercent over all patients (columns). Other proportions in table over disease category (rows).

${ }^{b}$ Diagnosis categorization is mutually exclusive. When bacteremia and source are both known, patient is characterized only as bacteremia.

antipseudomonal therapy past HD4, 45 (53\%) were deemed appropriate; appropriateness was determined based on antimicrobial susceptibility, neutropenia status, or culture-negative SSh. The 40 of 156 patients $(26 \%)$ who were not appropriately deescalated from antipseudomonal therapy highlight the need for ongoing antimicrobial stewardship.

\section{Sepsis Disease Severity}

Of the patients administered the SSP antibiotics, 13 (8\%) did not meet SIRS criteria, 6 (4\%) had SIRS or sustained organ dysfunction but were ultimately determined to have an alternative diagnosis, 72 (44\%) had sepsis, 46 (29\%) had SS, and 25 (15\%) had SSh (Table 2). Vasopressors were used in 11 (44\%) of those with SSh. The mean APACHE II score for patients without SS was 16 (SD, 4), with SS was 17 (SD, 4), and with SSh was 23 (SD, 8) (eFigure 1 ). Patients with more severe disease also received increasing 6- and 24-hour volumes of crystalloid infusion (eFigure 2). All patients with confirmed SS/SSh had at least one organ system with dysfunction, and 22 patients with only sepsis had a single documented hypotensive reading not considered sufficient criteria for SS (eAppendix 1 and eTable 4).

\section{Mortality and Time to Antibiotics}

Mortality was rare; only 1 person died during hospitalization, and mortality within 30 days occurred in only 6 of $160(3.8 \%$; 95\% CI, 1.4-8.0) persons not censored before 30 days. Of these, 2 had SS and 1 had SSh, for a $4.2 \%$ mortality rate $(95 \%$ CI, 0.9 $11.9)$ in 71 patients with SS/SSh.

The effect of time to antibiotic completion on mortality could not be sufficiently analyzed due to low mortality event rate. For each additional hour delay from the start of the clinical encounter until the completion of antibiotics, there was a $19 \%$ increased risk of developing SSh within 24 hours (95\% CI, 3\%-39\%; $P=.02$ ) and an $18 \%$ increased risk of developing SSh within 24 hours or death within 30 days (95\% CI, 4\%-34\%; $P=.01$ ). Results did not appreciably change with the parsimonious or maximally adjusted models (Table 3 ). Survival to 30 days could not be determined in 2 patients, and they were excluded from the model.

\section{Adverse Events}

Seventeen patients presented with or developed AKI during hospitalization; nephrotoxicity was unrelated to aminoglycoside in 10 patients, possibly related in 7 , and definitely related in none. Alternative causes for AKI included hypotension $(n=5)$, trimethoprim- 
Goldman et al

\begin{tabular}{|c|c|c|c|}
\hline $\begin{array}{l}\text { Model } \\
\text { Adjustment }\end{array}$ & Outcomes & $\begin{array}{l}\text { Relative Risk } \\
\quad(95 \% \mathrm{Cl})\end{array}$ & $\begin{array}{c}P \\
\text { Value }\end{array}$ \\
\hline \multicolumn{4}{|l|}{ Unadjusted } \\
\hline & 30-day mortality & $1.10(0.90-1.36)$ & .35 \\
\hline & SSh & $1.19(1.03-1.39)$ & .02 \\
\hline & $\begin{array}{l}\text { Either SSh or } \\
\text { 30-day mortality }\end{array}$ & $1.18(1.04-1.34)$ & .01 \\
\hline \multicolumn{4}{|c|}{ Adjusted for hematologic malignancy and race ${ }^{b}$} \\
\hline & 30-day mortality & $1.09(0.88-1.35)$ & .45 \\
\hline & SSh & $1.18(1.01-1.38)$ & .04 \\
\hline & $\begin{array}{l}\text { Either SSh or } \\
\text { 30-day mortality }\end{array}$ & $1.16(1.02-1.33)$ & .03 \\
\hline \multicolumn{4}{|c|}{$\begin{array}{l}\text { Adjusted for sex, race, hematologic malignancy, history of } \mathrm{HSCT} \text {, } \\
\text { receipt of dexamethasone }{ }^{c}\end{array}$} \\
\hline & 30-day mortality & $1.02(0.85-1.23)$ & .83 \\
\hline & SSh & $1.18(1.01-1.38)$ & .03 \\
\hline & $\begin{array}{l}\text { Either SSh or } \\
\text { 30-day mortality }\end{array}$ & $1.16(1.02-1.32)$ & .02 \\
\hline
\end{tabular}

Unadjusted and adjusted relative risk for each successive hour delay in time to receipt of third antibiotic for developing the outcomes of 30-day mortality, SSh, or the combined outcome of either 30-day mortality or SSh.

Abbreviations: HSCT, hematopoietic stem cell transplant; SSh, septic shock.

aAll models have the same number of 13 patients excluded due to any exposure, outcome or adjustment variable missing from the maximally adjusted model. Missing variables are: time to antibiotics $(n=2)$, survival to 30 days confirmed $(n=2)$, race $(n=9)$. bParsimonious multivariate model is adjusted for covariates associated with both the exposure (time to completion of antibiotics) and the combined outcome (SSh or cumulative 30-day mortality).

cMaximally adjusted multivariate model is adjusted for covariates associated with either the exposure (time to completion of antibiotics) or the combined outcome (SSh or cumulative 30-day mortality).

sulfamethoxazole $(n=1)$, and tacrolimus $(n=1$, elevated serum level to $25 \mathrm{ng} / \mathrm{mL}$ ). Rash was ascribed to SSP antibiotics in 6 cases and possibly related to SSP antibiotics in 10 cases. Other possibly related adverse events included itching with tobramycin and thrombocytopenia with linezolid. No cases of ototoxicity, Stevens-Johnson syndrome, toxic epidermal necrolysis, or serotonin syndrome were documented, and all cases of AKI and rash resolved.

\section{Microbiology}

Bacteremia occurred in 71 patients $(44 \%$; $95 \%$ CI, $36 \%-52 \%$ ), other infections without bacteremia in $33(20 \%)$, and culture-negative sepsis in 48 (30\%; eTable 5). Bacteremia was monomicrobial in 56 patients and polymicrobial in 15 patients, and no patients had fungemia. Highly pathogenic or in- trinsically resistant bloodstream microbial isolates included Pseudomonas aeruginosa (9 isolates), Acinetobacter baumannii (2 isolates), Enterobacter spp (11 isolates), and Stenotrophomonas maltophilia (3 isolates; eTable 6). No bloodstream isolate was considered a contaminant; in 13 cases in which coagulasenegative staphylococcus was isolated, all patients had either high-grade bacteremia (in $>1$ blood culture bottle), a central venous catheter site infection, or cellulitis. Of 93 unique isolates, 19 were DR, 1 was possible MDR, 13 were confirmed MDR, and 4 were possible XDR isolates.

A total of 90 (96.8\%; 95\% CI, 90.9\%-99.3\%) of 93 isolated bloodstream microbes were susceptible to $\geq 1$ SSP antibiotics administered (the "observed scenario"). The remaining 3 isolates were Stenotrophomonas maltophilia, which are intrinsically resistant to carbapenems and aminoglycosides. If IDSA guidelines $^{8}$ for antipseudomonal $\beta$-lactam monotherapy or combination with vancomycin as indicated were instead followed, these "hypothetical alternative scenarios" would result in less sufficient empiric coverage of the bacterial isolates. If piperacillin/ tazobactam was the administered alternative, 74 of 92 $(80.4 \%)$ microbial isolates would have been susceptible, 82 of 92 would have been susceptible $(89.1 \%)$ if carbapenem was administered, 78 of 91 (85.7\%) for cefepime, and 73 of 92 (79.3\%) for ceftazidime, with $P<.01$ for all comparisons with the observed scenario. Antipseudomonal cephalosporins are possible SSP alternatives to carbapenems (eFigure 3); however, 24 of 92 isolates (26\%) were nonsusceptible to all components in at least one hypothetical alternative scenario. Any choice of $\beta$-lactam monotherapy with the addition of vancomycin for indication would be inferior to the carbapenem, aminoglycoside, and oxazolidinone combination of the SSP for first-dose empiric therapy.

\section{Discussion}

Survival was excellent in this highly immunocompromised cohort of outpatients with cancer and sepsis treated with the SSP, including in the subset with SS/SSh. This novel care bundle is composed of 3 antibiotics (antipseudomonal carbapenem, tobramycin, and linezolid) that can be concurrently administered via rapid infusion (<30 minutes) with other supportive treatments. This outpatient cohort 
was not "good risk" simply due to ambulatory status; many had refractory hematologic malignancies or were highly immunocompromised with recent or current induction or conditioning chemotherapy, immunosuppressives, or high-dose corticosteroid therapy. APACHE II score in those with SS/SSh was similar to prior studies. ${ }^{16}$ Clinicians used the SSP to aggressively treat patients on presentation and before fully evaluating the severity of sepsis syndrome or definitely diagnosing bacteremia. We did not find nephrotoxicity attributed to a short course of tobramycin, an observation supported by recent retrospective studies ${ }^{17,18}$ and an animal model. ${ }^{19}$ Inappropriate use of initial empiric broad antibiotics occurred in only $8 \%$ of patients receiving the SSP. Hospital-based teams promptly de-escalated therapy from tobramycin and linezolid, but $26 \%$ of persons remained on anti-pseudomonal therapy inappropriately at HD4. The high prevalence of intrinsically DR and MDR bacteremia underscores the need for effective, initial broad coverage. Guideline-recommended empiric therapy ${ }^{8,20}$ would have been inactive for a considerable number of microbial isolates.

Epidemiology of SS/SSh in patients with cancer has only been studied in hospitalized patients, often in the intensive care unit. ${ }^{21}$ Mortality in these cohorts ranges from $34 \%$ to $69 \%^{1-3,17,22,23}$ (eTable 7). Compared against these data, the $4.2 \%$ mortality rate observed in the subset of patients with SS/SSh is considerably lower. Very conservative definitions of SS and SSh were applied to carefully extracted clinical data (eAppendix 1) making it unlikely that these patients had less severe disease compared with those reported on in prior studies. ${ }^{1-3,17,22,23}$ Although sepsis-related mortality is decreasing in patients with cancer ${ }^{17,22}$ and without, ${ }^{24}$ the excellent survival demonstrated in this cohort is unlikely to be due only to secular trends in the management of critically ill patients. Rather, we suggest that the low mortality seen in this cohort was due to standardization of effective antimicrobial therapy coadministered in rapid infusion with other supportive care through a simplified order bundle. These interventions were delivered early in the disease course before the development of multisystem organ failure, which is characteristic of untreated SS/SSh. Indeed, each hour delay to completion of antibiotics was associated with a statistically significant higher risk of developing SSh or 30-day mortality.
Bundled care for SS/SSh improves outcomes. ${ }^{25-27}$ The National Quality Forum recently ratified measures to assess a resuscitation bundle in patients with SS/SSh, and CMS required public reporting starting in October 2015.8 Our data validate this bundled approach to care, specifically for outpatients with cancer and SS/SSh, a high-risk group never previously studied.

Appropriate choice of empiric antibiotics for patients with cancer and SS/SSh is controversial. Meta-analyses in patients with cancer and neutropenic fever ${ }^{7}$ and patients without cancer who have sepsis $^{29}$ found no mortality difference between $\beta$-lactam monotherapy versus $\beta$-lactam plus aminoglycoside combination therapy, and increased nephrotoxicity with combination therapy. However, most of these patients did not have SS/SSh.,29 Limited data in patients without cancer suggest a mortality benefit to combination therapy in those with SS/SSh. ${ }^{30-32}$ A possible reason why data on combination therapy are conflicting is that antibiotics are often given sequentially, leading to infusion times of several hours, often after delays in initiation. Until now, the metric has been time to initiation of first antibiotic, ${ }^{5,6}$ instead of the perhaps more relevant time to completion of all components of the antibiotic infusion, as presented here. Antipseudomonal cephalosporins are possible SSP alternatives to carbapenems (eFigure 3). The analysis provided in the hypothetical alternative scenarios suggests that the triple combination of the SSP, as specified in the NCCN Guidelines, ${ }^{9}$ assures the broadest coverage for empiric first-dose therapy in unstable patients with cancer and presumed SS/ SSh.

Interpretations are limited by the lack of a contemporaneous control group or randomization to the SSP. Although we enrolled sequential patients, it is possible that potentially eligible patients who had SS/SSh did not receive the intervention and were hence not included in the cohort. Although the SSP was rolled out clinic-wide, patients might have been missed due to incomplete penetrance of the educational program, failure of clinicians to recognize SS/SSh, or subjective patient factors not easy to ascertain retrospectively. 
Goldman et al

\section{Conclusions}

Our results demonstrate excellent survival for patients with cancer and SS/SSh who are emergently administered a short course of broad-spectrum empiric antibiotics infused rapidly and concurrently with other resuscitative care. These data extend our knowledge of benefit for timely antibiotics to the outpatient setting and support use of short-course combination antibiotics for SS/SSh in patients with cancer. The strategy of rapid, concurrent antibiotic infusion initiated in the proximal phase of illness as empiric therapy should be studied in the inpatient setting. A multicenter trial is warranted in immunocompromised patients who develop SS/SSh, including those with cancer, solid organ transplant recipients, and patients receiving other iatrogenic immunosuppressive therapy.

\section{Acknowledgments}

The authors would like to thank the medical staff, especially the nurses, and the patients and their families. We thank Marco Mielcarek, Steve Fijalka, David Carlbom, and Claudia Stener for helpful discussions, and Christine Fong for supporting electronic database query.

\section{References}

1. Williams MD, Braun LA, Cooper LM, et al. Hospitalized cancer patients with severe sepsis: analysis of incidence, mortality, and associated costs of care. Crit Care 2004;8:R291-298.

2. Azoulay E, Mokart D, Pene F, et al. Outcomes of critically ill patients with hematologic malignancies: prospective multicenter data from France and Belgium-a groupe de recherche respiratoire en reanimation oncohematologique study. J Clin Oncol 2013;31:2810-2818.

3. Mokart D, Saillard C, Sannini A, et al. Neutropenic cancer patients with severe sepsis: need for antibiotics in the first hour. Intensive Care Med 2014;40:1173-1174.

4. Lin MY, Weinstein RA, Hota B. Delay of active antimicrobial therapy and mortality among patients with bacteremia: impact of severe neutropenia. Antimicrob Agents Chemother 2008;52:3188-3194.

5. Kumar A, Roberts D, Wood KE, et al. Duration of hypotension before initiation of effective antimicrobial therapy is the critical determinant of survival in human septic shock. Crit Care Med 2006;34:1589-1596.

6. Ferrer R, Martin-Loeches I, Phillips G, et al. Empiric antibiotic treatment reduces mortality in severe sepsis and septic shock from the first hour: results from a guideline-based performance improvement program. Crit Care Med 2014;42:1749-1755.

7. Paul M, Dickstein $Y$, Schlesinger A, et al. Beta-lactam versus beta-lactamaminoglycoside combination therapy in cancer patients with neutropenia. Cochrane Database Sys Rev 2013;6:CD003038.

8. Freifeld AG, Bow EJ, Sepkowitz KA, et al. Clinical practice guideline for the use of antimicrobial agents in neutropenic patients with cancer: 2010 update by the Infectious Diseases Society of America. Clin Infect Dis 2011;52:427431.

9. Baden LR, Swaminathan S, Angarone M, et al. NCCN Clinical Practice Guidelines in Oncology: Prevention and Treatment of Cancer-Related Infections. Version 2.2016. J Natl Compr Canc Netw 2016;14:882-913.

10. Perez F, Adachi J, Bonomo RA. Antibiotic-resistant gram-negative bacterial infections in patients with cancer. Clin Infect Dis 2014;59(Suppl 5):S335 339.

11. Vaughn JE, Othus M, Powell MA, et al. Resource utilization and safety of outpatient management following intensive induction or salvage chemotherapy for acute myeloid leukemia or myelodysplastic syndrome: a nonrandomized clinical comparative analysis. JAMA Oncol 2015;1:11201127.

12. Dellinger RP, Levy MM, Rhodes A, et al. Surviving sepsis campaign: international guidelines for management of severe sepsis and septic shock: 2012. Crit Care Med 2013;41:580-637.

13. Knaus WA, Draper EA, Wagner DP, Zimmerman JE. APACHE II: a severity of disease classification system. Crit Care Med 1985;13:818-829.

14. Magiorakos AP, Srinivasan A, Carey RB, et al. Multidrug-resistant, extensively drug-resistant and pandrug-resistant bacteria: an international expert proposal for interim standard definitions for acquired resistance. Clin Microbiol Infect 2012;18:268-281.

15. Leclercq R, Cantón R, Brown DF, et al. EUCAST expert rules in antimicrobial susceptibility testing. Clin Microbiol Infect 2013;19:141-160.

16. Rhodes A, Phillips G, Beale R, et al. The Surviving Sepsis Campaign bundles and outcome: results from the International Multicentre Prevalence Study on Sepsis (the IMPreSS study). Intensive Care Med 2015;41:1620-1628.

17. Legrand M, Max A, Peigne V, et al. Survival in neutropenic patients with severe sepsis or septic shock. Crit Care Med 2012;40:43-49.

18. Picard W, Bazin F, Clouzeau B, et al. Propensity-based study of aminoglycoside nephrotoxicity in patients with severe sepsis or septic shock. Antimicrob Agents Chemother 2014;58:7468-7474.

19. Lipcsey M, Carlsson M, Larsson A, et al. Effect of a single dose of tobramycin on systemic inflammatory response-induced acute kidney injury in a 6-hour porcine model. Crit Care Med 2009;37:2782-2790.

20. Baden LR, Bensinger W, Angarone M, et al. Prevention and treatment of cancer-related infections. J Natl Compr Canc Netw 2012;10:1412-1445.

21. Cohen J, Vincent JL, Adhikari NK, et al. Sepsis: a roadmap for future research. Lancet Infect Dis 2015;15:581-614.

22. Pene F, Percheron S, Lemiale $V$, et al. Temporal changes in management and outcome of septic shock in patients with malignancies in the intensive care unit. Crit Care Med 2008;36:690-696.

23. Larche J, Azoulay E, Fieux F, et al. Improved survival of critically ill cancer patients with septic shock. Intensive Care Med 2003;29:1688-1695.

24. Kaukonen KM, Bailey M, Suzuki S, et al. Mortality related to severe sepsis and septic shock among critically ill patients in Australia and New Zealand, 2000-2012. JAMA 2014;311:1308-1316.

25. Rivers E, Nguyen B, Havstad S, et al. Early goal-directed therapy in the treatment of severe sepsis and septic shock. N Engl J Med 2001;345:13681377.

26. Barochia AV, Cui X, Vitberg D, et al. Bundled care for septic shock: an analysis of clinical trials. Crit Care Med 2010;38:668-678

27. Levy MM, Rhodes A, Phillips GS, et al. Surviving Sepsis Campaign: association between performance metrics and outcomes in a 7.5-year study. Crit Care Med 2015;43:3-12.

28. Surviving Sepsis Campaign. Sepsis Measures Ratified by National Quality Forum. Available at: http://www.survivingsepsis.org/News/Pages/SepsisMeasures-Ratified-by-NQF.aspx. Accessed November 17, 2015.

29. Paul M, Silbiger I, Grozinsky $S$, et al. Beta lactam antibiotic monotherapy versus beta lactam-aminoglycoside antibiotic combination therapy for sepsis. Cochrane Database Sys Rev 2006:CD003344.

30. Kumar A, Safdar N, Kethireddy S, Chateau D. A survival benefit of combination antibiotic therapy for serious infections associated with sepsis and septic shock is contingent only on the risk of death: a meta-analytic/ meta-regression study. Crit Care Med 2010;38:1651-1664.

31. Kumar A, Zarychanski R, Light B, et al. Early combination antibiotic therapy yields improved survival compared with monotherapy in septic shock: a propensity-matched analysis. Crit Care Med 2010;38:1773-1785.

32. Diaz-Martin A, Martinez-Gonzalez ML, Ferrer R, et al. Antibiotic prescription patterns in the empiric therapy of severe sepsis: combination of antimicrobials with different mechanisms of action reduces mortality. Crit Care 2012;16:R223. 